\title{
New-onset status epilepticus in SARS-CoV-2 infection: a case series
}

\author{
Marco Belluzzo ${ }^{1}$ (D) Annacarmen $\mathrm{Nilo}^{2} \cdot$ Mariarosaria Valente $^{2,3} \cdot$ Gian Luigi Gigli ${ }^{2,3}$
}

Received: 30 June 2021 / Accepted: 4 August 2021 / Published online: 16 August 2021

(c) Fondazione Società Italiana di Neurologia 2021

\begin{abstract}
Background Neurological manifestations of COVID-19 infection are well recognized. Seizures and status epilepticus (SE) have been reported as possible manifestations and/or complications of SARS-CoV-2 infection at different disease stages, but few data are known about the type, severity, treatment response, and recurrence.

Methods Single-center retrospective case series.

Results This case series describes four COVID-19-positive patients admitted to an Italian University Hospital, who developed status epilepticus during the active phase of disease, independently from the severity of respiratory symptoms. Two of them presented a relapse after resolution of the acute viral infection, a feature that has not been previously reported.

Conclusions Although a possible association between SE and COVID-19 has been reported, the exact etiopathogenetic mechanism remains still not understood. Our series adds new insights to shed further light on this controversial issue.
\end{abstract}

Keywords Status epilepticus $\cdot$ SARS-CoV-2 infection $\cdot$ Epilepsy $\cdot$ EEG $\cdot$ Recurrent SE

\section{Introduction}

Status epilepticus (SE) is a rare manifestation of SARSCoV-2 infection, which can take place in various disease stages. Little is known about its features, therapeutic response, and implications on survival. Current information is mainly based on single case reports [1].

Here, we present the largest to date case series of de novo SE in COVID-19 patients, highlighting several characteristics previously unreported.

Marco Belluzzo and Annacarmen Nilo contributed equally to this work.

Marco Belluzzo

marco.belluzzo@hotmail.com

1 Neurology Unit, Department of Neurosciences, S. Maria Della Misericordia University Hospital, Piazzale S. Maria della Misericordia, 15, 33100 Udine, Italy

2 Clinical Neurology Unit, Department of Neurosciences, S. Maria Della Misericordia University Hospital, Udine, Italy

3 Department of Medicine, University of Udine Medical School, Udine, Italy

\section{Methods}

In this retrospective case series, we identified 4 patients admitted at our Neurological Unit dedicated to COVID19-positive subjects between October 1, 2020, and March 31,2021 . Informed consent was obtained from the patients' family.

All patients had long-term EEG monitoring, which were reviewed by two independent epileptologists. For the definition of nonconvulsive SE (NCSE), the Salzburg Criteria [2] were followed. Serum inflammatory markers with the inclusion of IL-6 and IL-8 levels were routinely assessed during hospital stay. Spinal tap was performed in three subjects; the remaining patient denied the consent. Cerebrospinal fluid (CSF) IL-6 and IL-8 levels were available for every patient, as well as SARS-CoV-2 RNA, while CSF IgG detection were not performed by our laboratory. Table 1 summarized main laboratory findings. All our patients were not candidate for COVID-specific therapies; all of them underwent only supportive therapies (oxygen therapy, steroids, and low molecular weight heparin). 
Table 1 Serum and CSF levels of cytokines

\begin{tabular}{|c|c|c|c|c|c|c|c|}
\hline Patients & Cytokines & $\begin{array}{l}\text { Serum concentration, } \\
\mathrm{pg} / \mathrm{mL} \text { (range) }\end{array}$ & Interpretation & $\begin{array}{l}\text { CSF concentration, } \\
\mathrm{pg} / \mathrm{mL} \text { (range) }\end{array}$ & Interpretation & $\mathrm{CSF} / \mathrm{serum}$ ratio & $\begin{array}{l}\text { CSF SARS- } \\
\text { CoV-2 } \\
\text { RNA }\end{array}$ \\
\hline Patient 1 & $\begin{array}{l}\text { IL-6 } \\
\text { IL-8 }\end{array}$ & $\begin{array}{l}11.8(0.8-6.4) \\
8.9(6.7-16.2)\end{array}$ & $\begin{array}{l}\uparrow \\
\text { Normal }\end{array}$ & $\begin{array}{l}5(2.1-9.6) \\
71.5(22.0-61.0)\end{array}$ & $\begin{array}{l}\text { Normal } \\
\uparrow\end{array}$ & $\begin{array}{l}0.42 \\
8.03\end{array}$ & Negative \\
\hline Patient 2 & $\begin{array}{l}\text { IL-6 } \\
\text { IL-8 }\end{array}$ & $\begin{array}{l}33.0(0.8-6.4) \\
12.3(6.7-16.2)\end{array}$ & $\begin{array}{l}\uparrow \\
\text { Normal }\end{array}$ & $\begin{array}{l}\text { n.a } \\
\text { n.a }\end{array}$ & $\begin{array}{l}\text { n.a } \\
\text { n.a }\end{array}$ & $\begin{array}{l}\text { n.a } \\
\text { n.a }\end{array}$ & n.a \\
\hline Patient 3 & $\begin{array}{l}\text { IL-6 } \\
\text { IL-8 }\end{array}$ & $\begin{array}{l}12.0(0.8-6.4) \\
38.3(6.7-16.2)\end{array}$ & $\begin{array}{l}\uparrow \\
\uparrow\end{array}$ & $\begin{array}{l}8.9(2.1-9.6) \\
64.9(22.0-61.0)\end{array}$ & $\begin{array}{l}\text { Normal } \\
\uparrow\end{array}$ & $\begin{array}{l}0.74 \\
1.69\end{array}$ & Negative \\
\hline Patient 4 & $\begin{array}{l}\text { IL-6 } \\
\text { IL-8 }\end{array}$ & $\begin{array}{l}24.3(0.8-6.4) \\
17.0(6.7-16.2)\end{array}$ & $\begin{array}{l}\uparrow \\
\uparrow\end{array}$ & $\begin{array}{l}1.7(2.1-9.6) \\
63.0(22.0-61.0)\end{array}$ & $\begin{array}{l}\downarrow \\
\uparrow\end{array}$ & $\begin{array}{l}0.07 \\
3.71\end{array}$ & Negative \\
\hline
\end{tabular}

n.a., not available; $C S F$, cerebrospinal fluid; $I L$, interleukin

\section{Case presentation}

\section{Patient 1}

An 81-year-old female, with multiple comorbidities including systemic hypertension and primary hyperparathyroidism, became COVID-19 positive as a consequence of disease spreading among her relatives. One week later, she developed fever and confusion. Three days after hospital admission she became suddenly unresponsive. No signs of respiratory dysfunction were detectable. Brain CT scan, blood tests, and lumbar puncture did not reveal anything significant, with the exception of a weak positivity for anti-Zic4 antibody in blood and cerebrospinal fluid (CSF) samples. EEG showed abundant 1- to 2.5-Hz generalized sharp/triphasic waves predominant on the left frontoparietal region, concerning for nonconvulsive SE (NCSE) (Fig. 1A). Brain MRI showed multiple $\mathrm{T}_{2} /$ FLAIR/DWI hyperintense areas over the middle cerebellar peduncles,
Fig. 1 EEG recordings of patient no. 1 (A) and patient no. 4 (B). Panel A shows slow background activity with 1 - to 2.5 $\mathrm{Hz}$ generalized sharp/triphasic waves predominant on the left fronto-parietal region. Panel B presents $2-\mathrm{Hz}$ sharp-waves and sharp-and-slow-wave complexes on the left parietal region with diffusion to ipsilateral temporal region. Longitudinal EEG montage according to the $10 / 20$ International System + ECG, LFF: $70 \mathrm{~Hz}$, HFF: $0.3 \mathrm{~Hz}$, sensitivity $100 \mu \mathrm{V} / \mathrm{mm}$

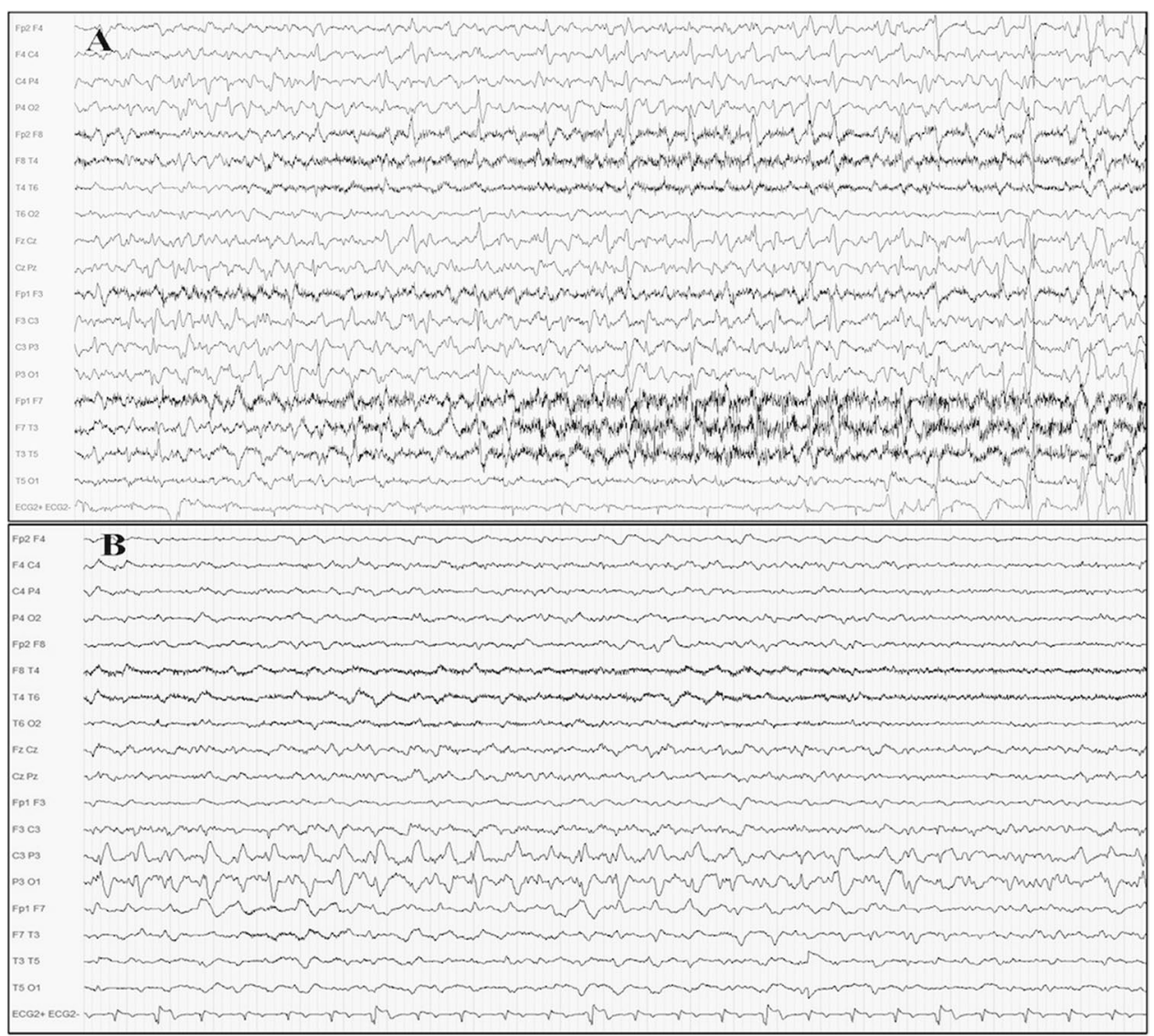


pons, basal ganglia, and bilateral frontal, temporal, and parietal subcortical white matter (Fig. 2A-C). The patient was given multiple ASMs: levetiracetam, valproic acid, brivaracetam. EEG monitoring did not document changes in the epileptic activity; therefore, steroid treatment followed by intravenous immunoglobulins and the adoption of ketogenic diet (ratio 4:1) was tried. With time, a progressive reduction of the epileptic activity and the appearance of a reactive theta $6-7-\mathrm{Hz}$ background rhythm were observed. After 2 months, a follow-up MRI showed a global reduction of the multiple hyperintense lesions (Fig. 2D-F). Despite these improvements, the patient remained drowsy, with a limited ability to interact with her surroundings and was eventually discharged to a nursing home for dependent people.

\section{Patient 2}

A 61-year-old female with complex comorbidities including obesity and left hemiplegia after rupture of a right internal carotid aneurysm presented with recurrent generalized tonic-clonic seizures. A brain CT scan did not show acute lesions, while lung CT scan documented a moderate degree of interstitial pneumonia and COVID-19 rapid testing was positive. Patient was intubated and sedated on propofol. An EEG demonstrated slow background activity with bursts of 3-Hz sharp-waves over the left hemisphere, interchanged to recruiting activity on the same region (Fig. 3A). The epileptic activity stopped after levetiracetam administration and the patient rapidly improved. During the rest of her hospital stay, she presented further two brief right motor seizures, prompting the introduction of lacosamide. After 14 days, she was discharged. However, 2 days later, she was readmitted owing to septic shock secondary to inhalation pneumonia. In spite of a prompt response to antibiotics and ventilatory support, the patient remained comatose. An EEG was performed, displaying continuous generalized 3-3.5-Hz sharpwaves, consistent with NCSE (Fig. 3B). Valproic acid and perampanel were unsuccessfully added to the pre-existent ASMs. Steroid and intravenous immunoglobulins were subsequently given, but the SE remained refractory. Twentyfive days after readmission, the patient's condition suddenly deteriorated due to nosocomial pneumonia and she expired on the following day.

\section{Patient 3}

A 63-year-old healthy woman was admitted to the hospital for fever, diarrhea, and hypotension. She was intubated due to respiratory distress. Serology test for SARS-CoV-2 antibodies was positive. A pulmonary CT scan showed diffuse ground-glass alterations consistent with COVID-19 pneumonia. She was weaned off mechanical ventilation and sedation 3 days later, but remained drowsy and unresponsive to
Fig. 2 Radiological findings of patient no. 1 with anti-Zic4 positivity. Brain magnetic resonance imaging (MRI): fluid-attenuated inversion recovery (FLAIR) images revealed hyperintensity in the middle cerebellar peduncles (A), pons (B), and bilateral basal ganglia (C). At 2-month follow-up, FLAIR hyperintensities globally reduced in the same regions $(\mathbf{D}$, E, F) with an increase of cortical atrophy
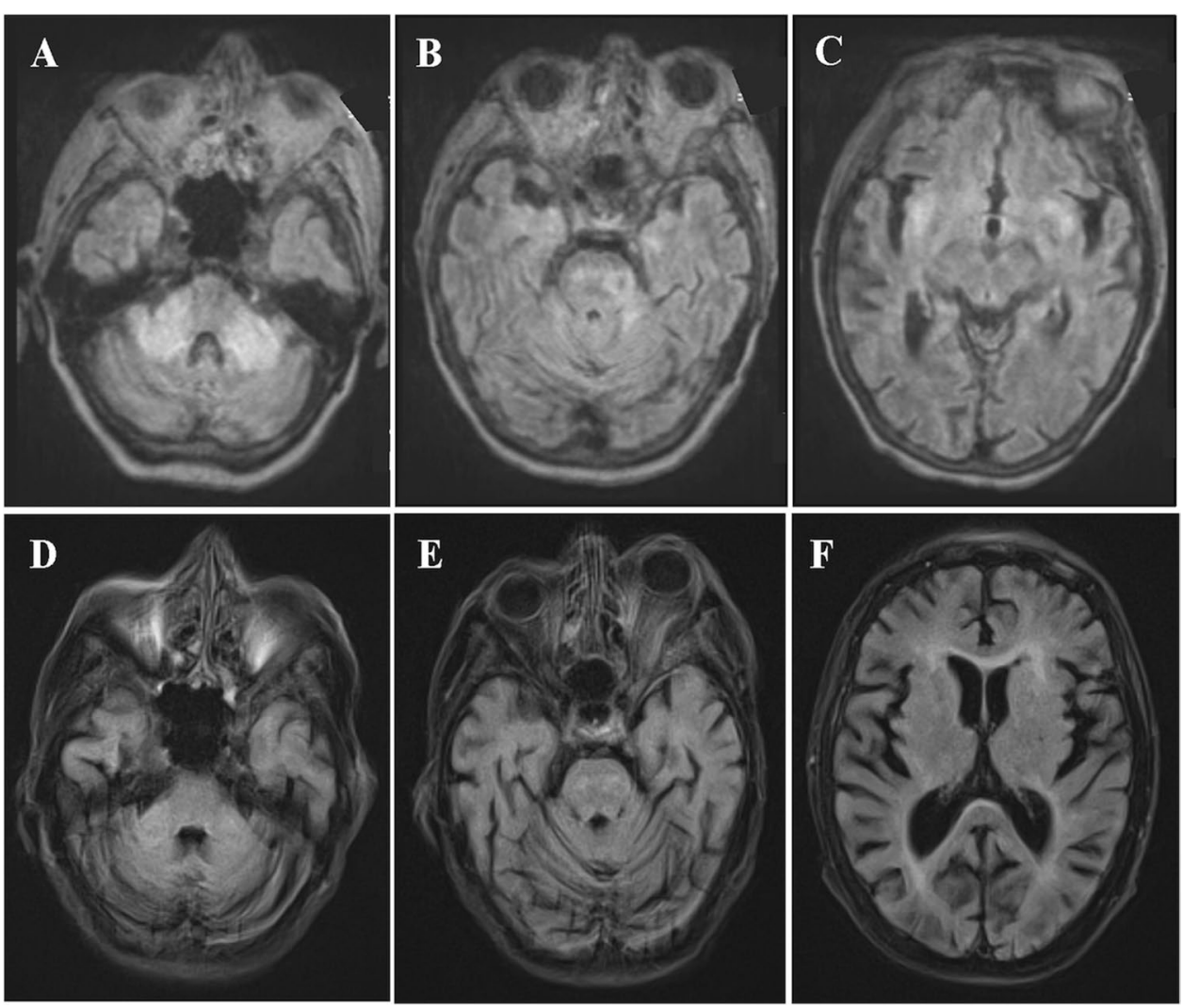


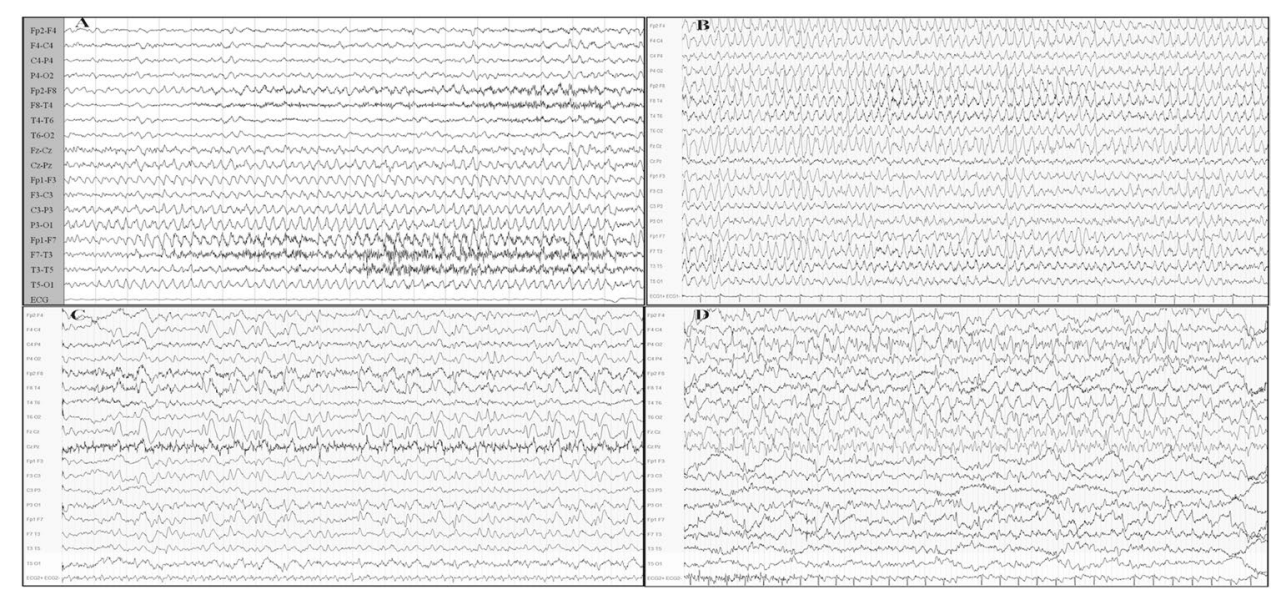

Fig. 3 EEG characteristics of patient no. 2 (A, B) and patient no. 3 (C, D). Patient no. 2: panel A shows slow background activity with continuous bursts of 3-Hz sharp-waves over the left hemisphere (first $\mathrm{SE}$ ); panel $\mathbf{B}$ demonstrates generalized $3-3.5-\mathrm{Hz}$ sharp-waves (SE relapse). Patient \#3: panel $\mathbf{C}$ shows diffuse slow background activity with $2-2.5-\mathrm{Hz}$ spike-and-wave and sharp-and-slow-wave complexes

external stimuli. An EEG showed diffuse slow background activity with $2-2.5-\mathrm{Hz}$ spike-and-wave and sharp-andslow-wave complexes over bilateral frontal regions with right prevalence, compatible with NCSE (Fig. 3C), which resolved 2 days after the introduction of levetiracetam. Brain MRI was unremarkable. The patient gradually improved and was discharged home without symptoms. After 11 days, she was referred again to the hospital for confusion, followed by a left motor seizure with bilateral tonic-clonic diffusion. Due to persistent disorientation, obsessive ideas, and left lower limb motor deficit, she performed an EEG that showed abundant and continuous $2-\mathrm{Hz}$ sharp-waves on the right parietal region with contralateral diffusion (Fig. 3D). An ensuing brain MRI showed DWI hyperintense lesions on the right cortical occipito-parietal and bilateral frontoparietal regions, with no contrast enhancement. CSF tests were unrevealing. Lacosamide was added on with resolution of motor signs, but with no effect on the neuropsychiatric symptoms. Therefore, levetiracetam was replaced with valproic acid together with a short course of dexamethasone, with rapid clinical and neurophysiological improvement. A new brain MRI obtained 16 days afterwards was negative. The patient was eventually discharged home without neurological symptoms.

\section{Patient 4}

An 82-year-old woman, with multiple comorbidities including systemic hypertension and moderate obesity, developed COVID-19 pneumonia with mild respiratory insufficiency and was admitted to a Covid Hospital Unit and treated with dexamethasone and supplemental oxygen. After 10 days, she over bilateral frontal regions with right prevalence (electrodes Fp2, F4, and F8) (first SE); panel D reports continuous 2-Hz sharp waves on the right parietal region with contralateral diffusion (SE relapse). Longitudinal EEG montage according to the 10/20 International System + ECG, LFF: $70 \mathrm{~Hz}$, HFF: $0.3 \mathrm{~Hz}$, sensitivity $100 \mu \mathrm{V} / \mathrm{mm}$

presented an episode characterized by aphasia and partial loss of awareness, lasting few minutes. A brain CT scan was unremarkable. She was discharged home without therapy, but 3 days later she was admitted again to the hospital for aphasia and disorientation. An EEG showed 2-Hz spikeand-wave and sharp-and-slow-wave complexes on the left temporo-parietal region (Fig. 1B). She was treated with lorazepam without effect; therefore, lacosamide was introduced, inducing a prompt resolution of the SE. Blood and CSF tests were unrevealing. A brain MRI showed a small right nucleo-capsular ischemic lesion. She did not present further seizures throughout hospitalization and remained asymptomatic until discharge.

\section{Discussion}

Since the onset of COVID-19 pandemic, a growing number of reports illustrate a wide range of neurological manifestations in the context of active or successive to SARS-CoV-2 infection, including seizures and SE $[1,3]$. Here, we detailed four COVID-19 cases who developed SE at some point of the active viral disease.

The potential etiology of epileptic manifestations in patients with COVID-19 are likely heterogeneous, including hypoxemia, metabolic derangements, direct viral injury, cytokine storming, and secondary autoimmune responses [4]. Central nervous system viral invasion is a controversial issue and it has been reported only in a very small minority of SE reports so far $[1,5]$. The para-/post-infectious explanation appears to be an enticing hypothesis: SE may share the same mechanisms of well-known post-COVID-19 
inflammatory diseases such as Guillain-Barré syndrome and acute disseminated encephalomyelitis, although definite evidence is lacking $[1,6,7]$.

SE occurrence and severity seem independent from the underlying respiratory manifestations and the severity of hypoxemia [1]. This apparent inconsistency may be explained by the fact that cytokine production can differ greatly in various bodily organs [8]. In fact, in our cases, we found elevated values of IL- 6 and also IL- 8 in the serum, while in CSF only IL- 8 was significantly increased in all three tested patients. As reported in other cases [9, 10], it is possible that IL-8 pathway can be relevant in the neuroinflammation response induced by SARS-CoV-2.

Regarding our small series, based on temporal evolution, laboratory, and MRI findings, SE in patient no. 4 can be reasonably ascribed to the intense inflammatory storm. Patient nos. 2 and 3 presented a short-term SE relapse, a feature previously unreported. Both had developed the first episode in the context of intense inflammatory response and marked respiratory syndrome; both SE resolved rapidly after ASMs starting. The relapse took place approximately 10 days later, in case no. 2 shortly after a secondary pneumonia, while in case no. 3 without clear precipitating factors. SE became refractory in patient no. 2 , while it rapidly improved after medications adjustment in case no. 3. The occurrence of a short-term relapse may suggest a para-infectious immune etiology by analogy with similar conditions such as acute inflammatory polyradiculoneuropathies, which in some cases present with one or multiple flares instead of the typical monophasic course. Furthermore, in one subject, the relapse was closely associated to an inhalation pneumonia, which might have acted as a booster for a waning yet still active aberrant immune response.

More problematic remains the interpretation of case no. 1 , in whom there were no respiratory symptoms. The identification of anti-Zic4 antibody in blood and CSF appears to be a novel finding. There have been few reports on the association of the anti-NMDAr antibody autoimmune syndrome and the SARS-CoV-2 infection [11, 12]. NMDAr encephalitis represents a frequent cause of autoimmune SE and sometimes viral infections may trigger its appearance [13]. Zic4 antibodies are instead associated to underlying tumors, tend to frequently co-occur with other antibodies, and usually relate to pure or predominant cerebellar syndromes [14]. No tumor was identified in our case, although some investigations were withheld given the unfavorable clinical conditions of the patient. A causal role of Zic4 antibody can be speculated based on the brain MRI findings and their evolution over time. However, it remains difficult to discern if the SE activation was due only to the inflammatory storm or to an immune-mediated mechanism induced by anti-Zic4 antibodies. We cannot exclude the possibility that Zic4 positivity was just the result of a non-specific wide immune activation.

\section{Conclusion}

SE associated to SARS-CoV-2 infection remains an elusive entity and its range of presentations and pathophysiology are yet to be fully disclosed. Our small series adds new insights to shed further light on this controversial argument.

Acknowledgements Data Access, responsibility, and analysis: all the authors had full access to all the data in the study and take responsibility for the integrity of the data and the accuracy of the data analysis.

Author contribution MB: conceptualization, methodology, data curation, data analysis and interpretation, writing-original draft preparation, writing (review and editing).

AN: conceptualization, methodology, data curation, data analysis and interpretation, writing-original draft preparation, writing (review and editing).

MV and GLG: supervision study, writing (review and editing).

All the authors approved the submitted version.

\section{Declarations}

Ethical approval The work described has been carried out in accordance with The Code of Ethics of the World Medical Association (Declaration of Helsinki) for experiments involving humans.

Informed consent Informed consent for data publication has been achieved.

Conflict of interest The authors declare that the research was conducted in the absence of any commercial or financial relationships that could be construed as a potential conflict of interest.

\section{References}

1. Dono F, Nucera B, Lanzone J, Evangelista G, Rinaldi F, Speranza $R$ et al (2021) Status epilepticus and COVID-19: a systematic review. Epilepsy Behav 118:107887. https://doi.org/10.1016/j. yebeh.2021.107887

2. Leitinger M, Beniczky S, Rohracher A, Gardella E, Kalss G, Qerama E et al (2015) Salzburg Consensus Criteria for NonConvulsive Status Epilepticus - approach to clinical application. Epilepsy Behav 49:158-163. https://doi.org/10.1016/j.yebeh. 2015.05.007

3. Paterson RW, Brown RL, Benjamin L, Nortley R, Wiethoff S, Bharucha $\mathrm{T}$ et al (2020) The emerging spectrum of COVID-19 neurology: clinical, radiological and laboratory findings. Brain 143(10):3104-3120. https://doi.org/10.1093/brain/awaa240

4. Vohora D, Jain S, Tripathi M, Potschka H (2020) COVID-19 and seizures: is there a link? Epilepsia 61(9):1840-1853. https://doi. org/10.1111/epi.16656

5. Karvigh SA, Vahabizad F, Mirhadi MS, Banihashemi G, Montazeri M (2021) COVID-19-related refractory status epilepticus with the presence of SARS-CoV-2 (RNA) in the CSF: a case report. Neurol Sci 15:1-4. https://doi.org/10.1007/s10072-021-05239-6

6. Carroll E, Neumann H, Aguero-Rosenfeld ME, Lighter J, Czeisler BM, Melmed K, Lewis A (2020) Post-COVID-19 inflammatory syndrome manifesting as refractory status epilepticus. Epilepsia 61(10):e135-e139. https://doi.org/10.1111/epi.16683

7. Manganotti P, Furlanis G, Ajcevic M, Moras C, Bonzi L, Pesavento V, Buoite Stella A. (2021) Intravenous immunoglobulin 
response in new-onset refractory status epilepticus (NORSE) COVID-19 adult patients. J Neurol 1-5. https://doi.org/10.1007/ s00415-021-10468-y

8. Wang C, Kang K, Gao Y, Ye M, Lan X, Li X et al (2020) Cytokine levels in the body fluids of a patient with COVID-19 and acute respiratory distress syndrome: a case report. Ann Intern Med 173(6):499-501. https://doi.org/10.7326/L20-0354

9. Pilotto A, Odolini S, Masciocchi S, Comelli A, Volonghi I, Gazzina S et al (2020) Steroid-responsive encephalitis in coronavirus disease 2019. Ann Neurol 88(2):423-427. https://doi.org/ 10.1002/ana.25783

10. Gigli GL, Vogrig A, Nilo A, Fabris M, Biasotto A, Curcio F et al (2020) HLA and immunological features of SARS-CoV-2-induced Guillain-Barré syndrome. Neurol Sci 41(12):3391-3394. https:// doi.org/10.1007/s10072-020-04787-7

11. Monti G, Giovannini G, Marudi A, Bedin R, Melegari A, Simone AM et al (2020) Anti-NMDA receptor encephalitis presenting as new onset refractory status epilepticus in COVID-19. Seizure 81:18-20. https://doi.org/10.1016/j.seizure.2020.07.006

12. Panariello A, Bassetti R, Radice A, Rossotti R, Puoti M, Corradin M, Moreno M, Percudani M (2020) Anti-NMDA receptor encephalitis in a psychiatric Covid-19 patient: a case report. Brain Behav Immun 87:179-181. https://doi.org/10.1016/j.bbi.2020.05. 054

13. Dalmau J, Armangué T, Planagumà J, Radosevic M, Mannara F, Leypoldt $F$ et al (2019) An update on anti-NMDA receptor encephalitis for neurologists and psychiatrists: mechanisms and models. Lancet Neurol 18(11):1045-1057. https://doi.org/10. 1016/S1474-4422(19)30244-3

14. Bataller L, Wade DF, Graus F, Stacey HD, Rosenfeld MR, Dalmau J (2004) Antibodies to Zic4 in paraneoplastic neurologic disorders and small-cell lung cancer. Neurology 62(5):778-782. https://doi. org/10.1212/01.wnl.0000113749.77217.01

Publisher's note Springer Nature remains neutral with regard to jurisdictional claims in published maps and institutional affiliations. 